\title{
An Evolutionary Perspective on Cancer, with Applications to Anticancer Drug Resistance Modelling and Perspectives in Therapeutic Control
}

\author{
Jean Clairambault* \\ Sorbonne Université, CNRS, Université de Paris, Laboratoire Jacques-Louis Lions \\ (LJLL ), E Inria Mamba team, Paris, France.
}

Received January 24, 2019; Accepted May 28, 2019

\begin{abstract}
The question of a mathematical representation and theoretical overcoming by optimised therapeutic strategies of drug-induced drug resistance in cancer cell populations is tackled here from the point of view of adaptive dynamics and optimal population growth control, using integro-differential equations. Combined impacts of external continuous-time functions, standing for drug actions, on targets in a plastic (i.e., able to quickly change its phenotype in deadly environmental conditions) cell population model, represent a therapeutical control to be optimised. A justification for the introduction of the adaptive dynamics setting, retaining such plasticity for cancer cell populations, is firstly presented in light of the evolution of multicellular species and disruptions in multicellularity coherence that are characteristics of cancer and of its progression. Finally, open general questions on cancer and evolution in the Darwinian sense are listed, that may open innovative tracks in modelling and treating cancer by circumventing drug resistance. This study sums up results that were presented at the international NUMACH workshop, Mulhouse, France, in July 2018.
\end{abstract}

AMS subject classifications: 35B40, 35Q92, 35R09, 45J05, 49J20, 92C50, 92D15, 92D25, 93D20

Key words: Integro-differential equations, asymptotic analysis, optimal control, mathematical oncology.

\section{Introduction}

This study presents an evolutionary viewpoint on cancer, seen as the 2 time scales of (large-time) evolution in the genomes and of (short-time) evolution in the epigenetic landscape of a constituted genome, which led to the proposal of mathematical models elaborated within the Jacques-Louis Lions laboratory of Sorbonne Université, Paris. These

${ }^{*}$ Corresponding author. Email address: jean. clairambault@inria.fr (J. Clairambault) 
views, inspired by works of Lineweaver, Davies and Vincent (cancer as anatomically located backward evolution in multicellular organisms, aka atavistic theory of cancer) and of Sui Huang and collaborators (revisited Waddington epigenetic landscape), respectively, may serve as guidelines to propose a global conception of cancer, including towards possible innovating therapeutic strategies.

Drug-induced drug resistance in cancer, the biological and medical question we are tackling from a theoretical point of view, may be due to biological mechanisms of different natures, mere local regulation, epigenetic modifications (reversible, nevertheless heritable) or genetic mutations (irreversible), according to the extent to which the genome of the cells in the population is affected. In this respect, the modelling framework of adaptive dynamics presented here is likely to biologically correspond to epigenetic modifications, although eventual induction of emergent resistant cell clones due to mutations under drug pressure is not to be excluded. From the biologist's point of view, we study phenotypically heterogeneous, but genetically homogeneous, cancer cell populations under stress by drugs.

The built-in targets for theoretical therapeutic control present in the phenotypestructured PDE models we advocate are not supposed to represent well-defined molecular effects of the drugs in use, but rather functional effects, i.e., related to cell death (cytotoxic drugs), or to proliferation in the sense of slowing down the cell division cycle without killing cells (cytostatic drugs). I propose that cell life-threatening drugs (cytotoxics) induce by far more resistance in the highly plastic cancer cell populations than drugs that only limit their growth (cytostatics), and that a rational combination of the two classes of drugs - and possibly others, adding relevant targets to the model - may be optimised to propose therapeutic control strategies to avoid the emergence of drug resistance in tumours.

We address this optimal control problem in the context of two populations, healthy and cancer, both endowed with phenotypes evolving under drug pressure acting as an environmental constraint, and reciprocally inhibiting the proliferation of the other population in a non-local Lotka-Volterra model. Our objective is thus to minimise the proliferation of a cancer cell population while limiting the emergence of drug resistance in it, and taking into account the constraint of limiting toxicity to a population of healthy cells, that are also targets of unwanted adverse effects of the cytotoxic drug.

To conclude, I present an informal list of open questions on cancer and its treatments that may be considered as challenges to mathematicians (and others).

\section{Biological background on evolution and cancer}

\subsection{Motivation from, and focus on, drug resistance in cancer}

Intra-tumour heterogeneity with respect to drug resistance potential, modelling betweencell phenotypic variability within cancer cell populations, is a convenient setting to represent continuous evolution towards drug resistance in tumours [6]. Going beyond the 
small scale of a human life, I will consider slow genetic mechanisms of 'the great evolution' that has designed multicellular organisms (see Figure 1), in the frame of which fast reverse evolution on smaller time windows, at the scale of a human disease, may explain transient or established drug resistance. The idea of cancer as a backwards evolution towards unicellularity, although not new [2,23], has recently be popularised as the atavistic theory of cancer, with many recent developments $[4,8,10,43,44,48]$.

A key concept to be added to reverse evolution, i.e., to the atavistic hypothesis, is the high plasticity shown by cancer cells, due to epigenetic (much faster than genetic mutations, and reversible) propension to reversal to a stem-like, de-differentiated (hence plastic) status, resulting in fast adaptability of cancer cell populations, makes them amenable to resist abrupt drug insult as response to extreme cellular stress $[15,16]$.

Such plasticity, itself reversible according to environmental pressure or relaxation, is captured by mathematical models that incorporate between-cell heterogeneity by making use of continuous phenotypic variables structuring the population $[29,30]$. Such models have the advantage of being compatible with optimal control methods for the theoretical design of optimised therapeutic protocols involving combinations of cytotoxic and cytostatic ( and later epigenetic?) treatments [39].

\subsection{Drug resistance: a phenomenon common to various therapeutic situations}

In therapeutic situations where an external pathogenic agent is proliferating at the expense of the resources of an organism: antibiotherapy, virology, parasitology, target populations are able to develop drug resistance mechanisms (e.g., expression of $\beta$-lactamase in bacteria exposed to amoxicillin). In cancer, there is no external pathogenic agent (even though one may have favoured the disease) and the target cell populations share much of their genome with the host healthy cell population, making overexpression of natural defence phenomena easy (e.g., ABC transporters in cancer cells).

Drug resistance may account for unexpected failures in targeted therapies, as they are meant to block a given intracellular (or intercellular) signalling pathway, very often related to proliferation, that can be coped with by adaptable, plastic cancer cells [14]. Note that drug resistance (and resistance to radiotherapy) is one of the many forms of fast resistance to cellular stress, possibly coded in 'cold', i.e., strongly preserved throughout evolution, rather than in 'hot', i.e., mutation-prone, genes [50].

Overexpression of $\mathrm{ABC}$ transporters [18], of drug processing enzymes, decrease of drug cellular influx, etc. [17] are relevant to describe molecular resistance mechanisms at the single cell level. However, at the cell population level, representing drug resistance by a continuously evolving abstract variable $x$ standing for the expression of a phenotype of resistance to a given cytotoxic drug is adapted to describe in a one-dimensional way evolution from total sensitivity $(x=0)$ towards total resistance $(x=1)$, and such abstract variable could be partly identified by measuring the expression of $A B C$ transporters such as P-glycoprotein (aka MDR1) [37].

Is such evolution towards drug resistance due to sheer Darwinian selection of the 


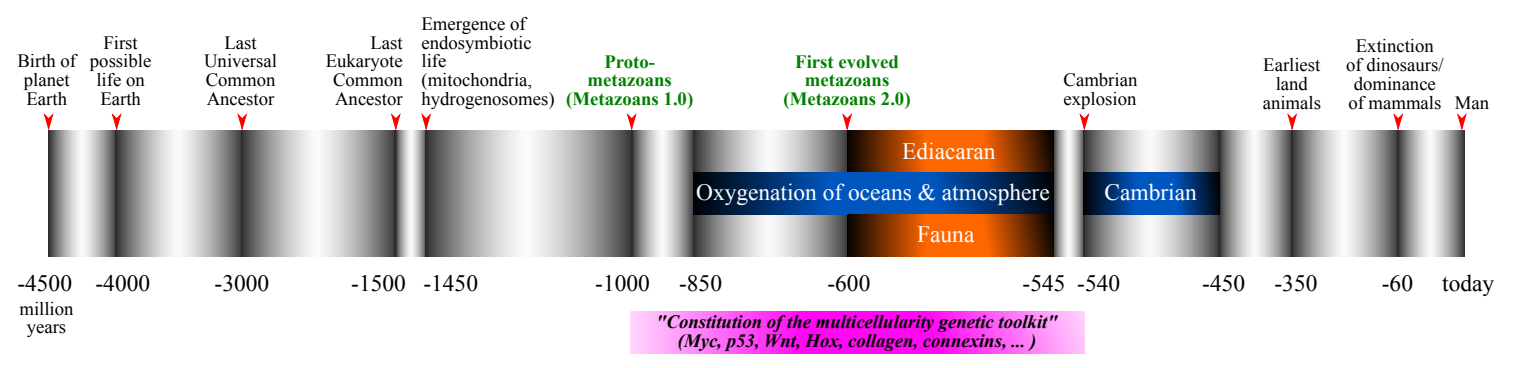

Figure 1: Schematic timeline of the evolution of life on Earth, emphasising emergence of multicellularity between -1000 and -500 million years from now (taken from [6]).

fittest by mutations in differentiation at cell division or, at least partially, due to phenotype adaptation in individual cells at the epigenetic level of the chromatin? A unique answer to this question does not result clearly from biological experiments, and indeed observations exist, showing that non-Darwinian phenomena, sometimes elicited to be of epigenetic nature, may explain such reversible evolution towards drug resistance in cancer cell populations [41].

\subsection{A possible evolutionary framework (long-term view): the atavistic hypothesis of cancer}

"Nothing in biology makes sense except in the light of evolution" [11]

"Cancer: more archeoplasm than neoplasm" [48]

The atavistic hypothesis of cancer $[4,8,10,43,44,48]$ states that cancer is a coarse, primitive state of multicellularity development that nevertheless involves all our genome. In this way, cancer represents a step in the reversion of the evolution of multicellular organisms towards (but not until) unicellular life.

A reasonable hypothesis, coming from the observation that cancer is a disease of the control and coherence of multicellularity, is that the genes that have appeared in the process of development to multicellularity are precisely those that are altered in cancer [10]. In the framework of cancer evolution in a single organism, in what order do these genes come altered? Admitting that the building of multicellularity starts in assemblies of cells by developing gene expression from 1) proliferation+apoptosis to 2) cell differentiation +division of work, and to 3) epigenetic control of differentiation and proliferation, this question is partially answered in [19] about acute myeloid leukaemia (AML) by phylogenetic studies on fresh blood samples from patients: it is likely to be, in the standard scenario in which p53 is not mutated (case of most AMLs), the reverse order, i.e., firstly mutation of an epigenetic control gene, then of a transcription factor corresponding to differentiation, and, only finally in this phylogeny, of a gene controlling proliferation.

Identifying those genes is of high interest, as reconstituting the phylogeny of this 
'multicellularity toolkit' $[12,13,52]$ should shed light on the robustness or fragility of genes (in a given organism) that have been altered in cancer. According to the atavistic hypothesis [10], cancer is a 'backward evolution' from a sophisticated form of multicellularity (us), in which epigenetic processes control gene regulatory networks of transcription factors: differentiation factors, p53, etc., that themselves physiologically control the basis of cellular life: proliferation. Following this point of view, i.e., that building multicellularity from coarse (proliferation) to fine (epigenetic control of differentiation) mechanisms, it is clear indeed that attacking cancer on proliferation is precisely attacking its most robust basis. It would be better to attack its weaknesses (e.g., absence of adaptive immune response) [26].

Furthermore, we bear in our genomes many attempts of species evolution since billions of years; dead-end tracks ('unused attractors' in S. Huang's and S. Kauffman's version of the Waddington landscape, cf. infra [21,22]) have been normally barred by Darwinian selection (e.g., silenced by epigenetic enzymes, resulting in evolutionary barriers in this landscape), but are still there, waiting to be triggered by the pressure of a changing environment. In cancer, global regulations (such as coherence messages from a central circadian clock) are lost, differentiation is out of control, so that, without regulation, local proliferations overcome; sophisticated adaptive epigenetic mechanisms are present, not controlling proliferation, but serving it (possibly recruited in the context of extreme cellular stress by stochastic or systematic expression of so-called cold genes? [50]).

Primitive forms of cooperation between specialised cells in a locally organised multicellular collection (tumour), with much plasticity among them, may be present, exhibiting coherent intratumoral heterogeneity, and escaping external control [31,36, 42]. Tumours are thus Metazoa 1.0 [10]. The basic cancer cell, be it isolated or included in a tumour is thus highly plastic (undifferentiated or able to easily dedifferentiate) and highly capable of adaptation to a hostile environment, as were its ancestors in a remote past of our planet (poor $\mathrm{O}_{2}$, acidic environment, high UV radiations,...) [10] and likely presently even more.

\subsection{Another evolutionary framework (life-term view): revisiting the Waddington epigenetic landscape}

"Nothing in evolution makes sense except in the light of systems biology" (S. Huang, 2012)

At the time scale of the development of a human life, for a given subject, the relevant time evolution is the one of a coherent succession of differentiations from one primitive cell towards a completely mature multicellular organism. This differentiation process, consisting of epigenetic modifications (aka epimutations) within the same genome, is metaphorically represented by the classic Waddington epigenetic landscape [49], illustrated on Figure 2, later revisited by Sui Huang [21,22], illustrated on Figure 3.

When the coherence in the multicellular design of an individual is altered by blockade of cell differentiation at a given stage and in a given organ, with maintained proliferation, 


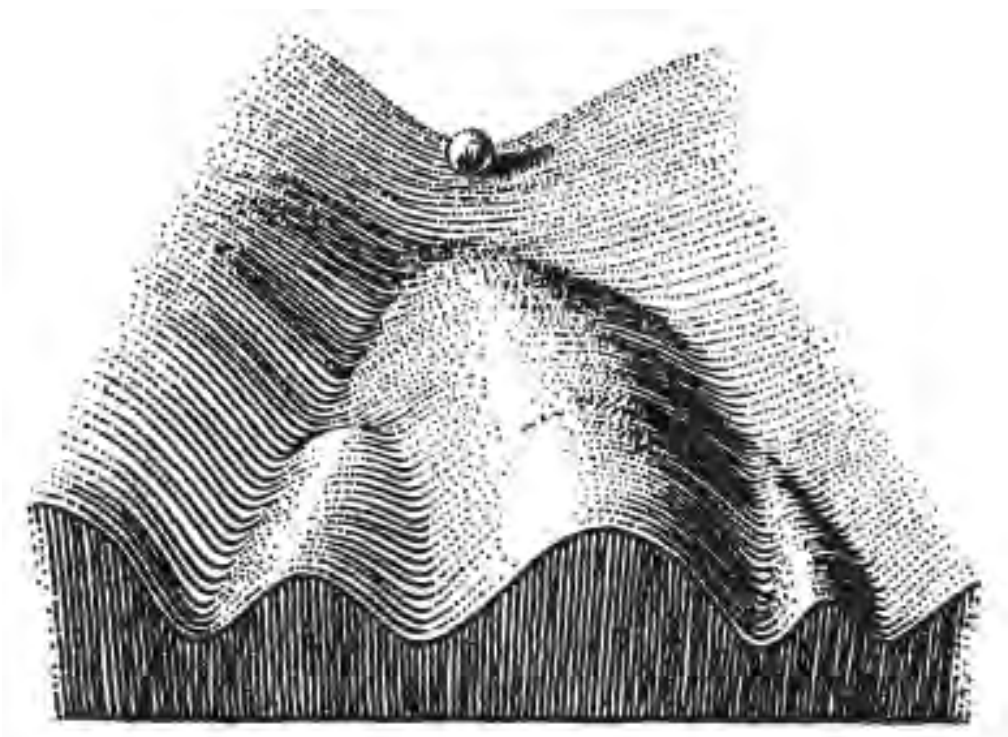

Figure 2: The classic Waddington landscape (1957) for cell differentiation [49].

in one of what $\mathrm{S}$. Huang calls unused attractors in the Waddington epigenetic landscape, see Figure 3, then can locally begin cancer. Such defect in local differentiation control may be due to mutations of epigenetic control genes (e.g., in the case of acute myeloid leukaemia (AML), TET2, DNMT3A, leading in a process that may take years, from absolute clinical and biological silence in early infancy, to the clinical manifestation of an $\mathrm{AML}$, in an elderly patient). It can also be due to the local action of external carcinogenic agents, including anticancer drugs that destabilise the genome, or to random mutations (less likely, and these can also result from prior epimutations [51]).

From the point of view of this metaphoric landscape, the plasticity of cancer cell populations can be seen as plasticity of the landscape (somehow from a rigid plaster-like to a smooth plasticine-like construction, erasing epigenetic barriers between valleys and (since the third dimension in the scenery represents differentiation potential of an initial totipotent cell) thoroughly flattening the whole landscape, resulting in a more and more poorly differentiated and incoherent multicellular ensemble of cells.

\section{Cell population adaptive dynamics}

\subsection{First model with (epi)mutation kernel, healthy and cancer cell population [29]}

Triggered by the fact that drug-induced drug resistance remains one of the main pitfalls and causes of death in the clinic of cancers, with the aim to later optimise drug delivery 


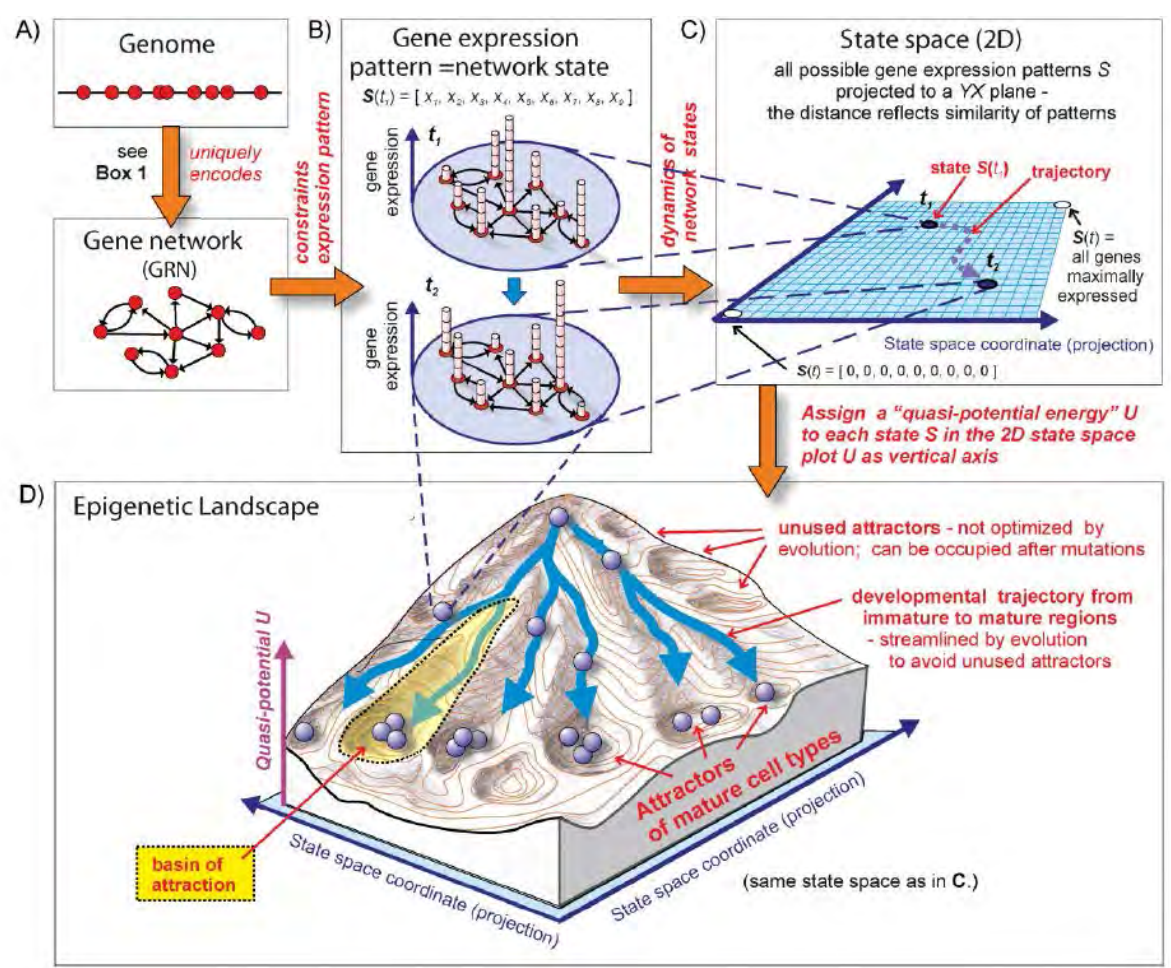

Figure 3: The Waddington landscape revisited by Sui Huang $(2011,2013)[21,22]$.

strategies to circumvent it, we proposed a drug resistance phenotype-structured model in which the phenotype $x \in[0,1]$ (from 0 , total sensitivity, to 1 , total resistance) was present in 4 terms: the basic proliferation rate $r$, the basic (without drugs added) death rate $d$, a drug sensitivity term $\mu_{C}$, and an integral term representing small mutations or epimutations, i.e., small changes in phenotype.

This first integro-differential model structured in a continuous trait $x$ representing the level of expression of a drug resistance phenotype (to a given drug), with variables representing the evolution of two cell populations, healthy and cancer, $n_{H}(x, t), n_{C}(x, t)$ being the densities of these cell populations ( $H=$ healthy, $C=$ tumour) runs as

(Resistant cancer case)

$$
\begin{gathered}
\frac{\partial}{\partial t} n_{C}(x, t)=[\overbrace{\left(1-\theta_{C}\right) r(x)}^{\text {growth }}-\overbrace{d(x)}^{\text {death }}-\overbrace{u(t) \mu_{C}(x)}^{\text {drugeffect }}] n_{C}(x, t) \\
+\theta_{C} \overbrace{\int r(y) M_{\sigma_{C}}(y, x) n_{C}(y, t) d y,}^{\text {birth with mutation }}
\end{gathered}
$$



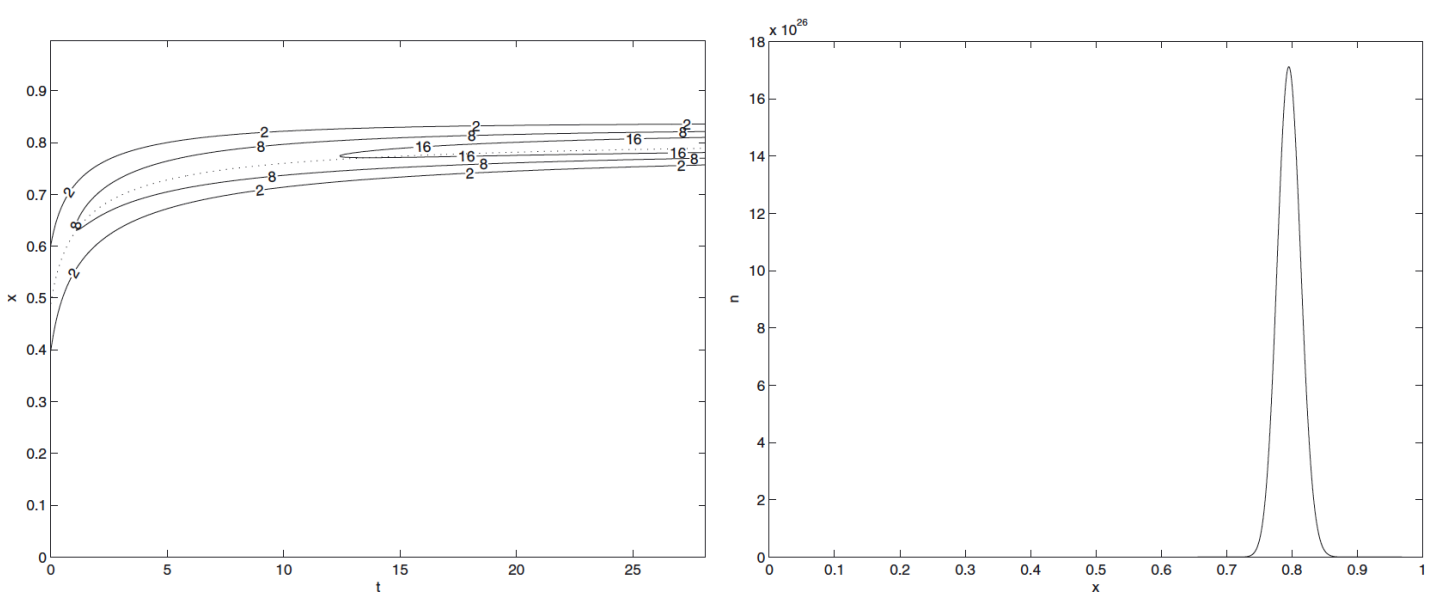

Figure 4: Resistant cancer cell population case. Left panel: starting from an arbitrary median phenotype $x=0.5$, level sets of a drug-resistant population in the $(t, x)$ (time and phenotype) plane. Right panel: asymptotic distribution of this drug-resistant population according to the drug resistance phenotype $x \in[0,1]$. [Resistant cancer cell population case: illustration of Gause's exclusion principle] here with $\theta_{C}=0, \mu_{C}(\cdot)>0, r^{\prime}(\cdot)<0, \mu_{C}^{\prime}(\cdot)<0$ (costly drug-induced resistance), with $u(t)=$ Cst [29].

where $r(x)=$ basic reproduction rate, $d(x)=$ basic death rate; we assume that

$$
r(0)>d(0)>0, \quad r^{\prime}(\cdot)<0, \quad r(1)=0, \quad d^{\prime}(\cdot)>0 .
$$

Here $\theta_{H}, \theta_{C} \quad\left(1>\theta_{C}>>\theta_{H} \geq 0\right)$ are the proportions of divisions with mutations; $\mu_{[H, C]}(x)\left(\right.$ with $\left.\mu_{C}^{\prime}(\cdot)<0\right)$ represents the phenotype-dependent response to cytotoxic drug, with concentration $u(t)$, designed to target cancer cells.

The assumptions $r(\cdot)>0, \mu_{C}(\cdot)>0, \mu_{C}^{\prime}(\cdot)<0$ and $r^{\prime}(\cdot)<0$ (cost of resistance: the higher is $x$, the lower is proliferation) represent the effect of drug resistance on the proliferation and death terms, plus an evolutionary double bind on resistant cancer cell populations, i.e., a trade-off between growing (thus getting exposed) and keeping still (thus surviving).

Here $u(t)$ denotes the instantaneous dose (concentration) of chemotherapy, only environmental pressure considered in this model. We assume that its effect is cytotoxic, i.e., on the death term only.

We then have the

Theorem 3.1. ([29]) (Resistant cell population case) There is monomorphic evolution of the cell population towards drug-induced drug resistance (illustrated on Figure 4).

In the same way, we represent the sensitive (or healthy) population case (same notations):

(Sensitive cancer - or healthy - case) 

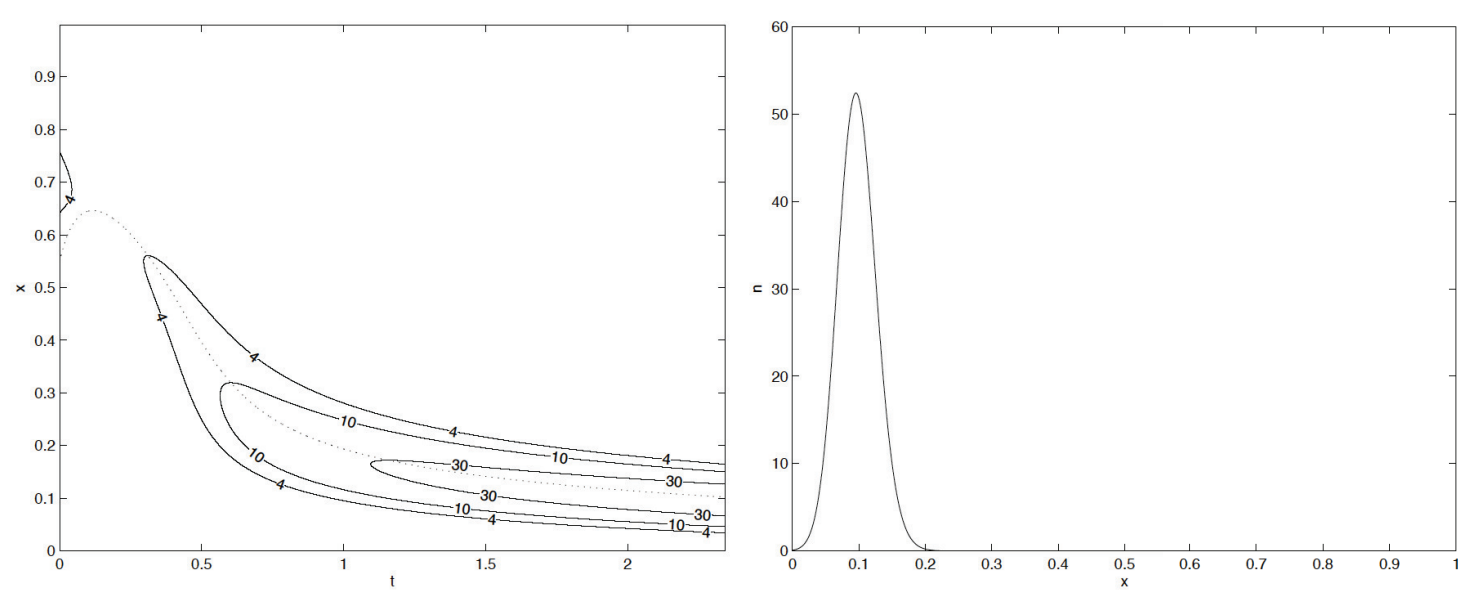

Figure 5: Healthy (or sensitive) cell population case. Left panel: starting from an arbitrary median phenotype $x=0.5$, level sets of a drug-sensitive population in the $(t, x)$ plane (time in abscissae and phenotype in ordinates). Right panel: asymptotic distribution of this population according to the drug resistance phenotype $x \in[0,1]$. [Sensitive (or healthy) cell population case: again, illustration of Gause's exclusion principle] here with $\theta_{H}=0$ (no mutations) and $\mu_{H}=0$ (no drug-induced resistance), with $u(t)=$ Cst [29].

$$
\begin{aligned}
\frac{\partial}{\partial t} n_{H}(x, t)= & {[\overbrace{\frac{1-\theta_{H}}{(1+\rho(t))^{\beta}} r(x)}^{\text {growth with homeostasis }}-\overbrace{d(x)}^{\text {death }}-\overbrace{u(t) \mu_{H}(x)}^{\text {drug effect }}] n_{H}(x, t) } \\
& +\frac{\theta_{H}}{(1+\rho(t))^{\beta}} \overbrace{\int r(y) M_{\sigma_{H}}(y, x) n_{H}(y, t) d y,}^{\text {birth with mutation }}
\end{aligned}
$$

where the total population is defined as $\rho(t)=\rho_{H}(t)+\rho_{C}(t)$, in which

$$
\rho_{H}(t)=\int_{x=0}^{\infty} n_{H}(x, t) d x, \quad \rho_{C}(t)=\int_{x=0}^{\infty} n_{C}(x, t) d x .
$$

( $\beta>0$ imposes healthy tissue homeostasis).

We then have the

Theorem 3.2. ([29]) (Sensitive cell population case) There is monomorphic evolution of the cell population towards drug sensitivity (illustrated on Figure 5).

However, whatever the qualities of this model, and its amenability to be analysed mathematically, we found out that the integral term resulted in a negligible part to the evolution of the trait in the population, and that almost all was due to the sensitivity term $\mu_{C}$ in the resistant cancer case. We thus reverted to another form of model, still nonlocal, but of the more classical Lotka-Volterra form, in which nonlocality is located in the logistic term, that represents limitation of growth by competition of every cell with 
all the others in the population, e.g., for space and nutriments. Note that an important characteristic of these integro-differential models, from a biological point of view, is that their evolution is totally reversible, i.e., if the environmental pressure represented by the drug infusion is withdrawn, then the model reverts to its drug-free status, and this is observed indeed in phenomena of acute drug resistance in cancer cell populations [41].

\subsection{Simple phenotype-structured population dynamics}

In the most general case, we aim at the description of the evolution of a population in time $t$ and in relevant phenotype $x$, where the 'structure variable' $x$ is a trait chosen as bearing the biological variability at stake, e.g. here (but many other instances may exist, such as cell size, age in cell division cycle, fecundity, viability, motility, etc., depending on the context), a resistance trait.

The variable at stake, $n(t, x)$, represents a population density of individuals bearing trait $x$ at time $t$. We investigate

(1) the evolution in numbers of individuals constituting the population

$$
\left.t \mapsto \rho(t)=\int_{0}^{1} n(t, x) d x \quad \text { (if, e.g., } x \in[0,1]\right),
$$

(2) the asymptotic distribution of the trait in the population

$$
x \mapsto \lim _{t \rightarrow+\infty} \frac{n(t, x)}{\rho(t)} .
$$

In the case of cancer cell populations:

(1) allows to study tumour growth;

(2) allows to study the evolution and asymptotic distribution of the trait.

Note that space is not necessarily a relevant structure variable when studying drug control.

We then propose a nonlocal (it is the logistic term which is nonlocal) Lotka-Volterra model: The variable $n(t, x)$ being the density of cells of trait phenotype $x \in[0,1]$ at time $t$

$$
\frac{\partial n}{\partial t}(t, x)=(r(x)-d(x) \rho(t)) n(t, x),
$$

with

$$
\rho(t):=\int_{0}^{1} n(t, x) d x \text { and } n(0, x)=n^{0}(x) .
$$

We assume reasonable $\left(C^{1}\right)$ hypotheses on $r$ and $d$, and $n^{0} \in L^{1}([0,1])$.

We then tackle the two questions above: what is the asymptotic behaviour of

(1) the total population $\rho(t)$ ? 


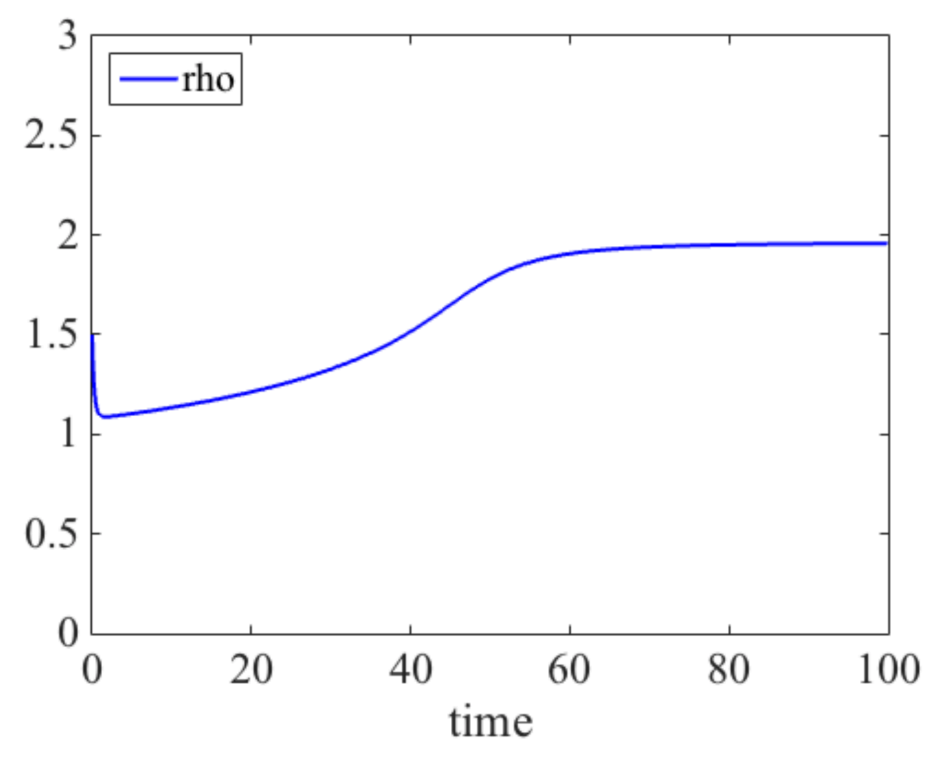

Figure 6: Convergence (one-population case): plot of $t \mapsto \rho(t)$.

(2) the distribution of the phenotype $x$ in the population (i.e., limit of $\frac{n(t, \cdot)}{\rho(t)}$ in $\left.M^{1}(0,1)\right)$ ?

Firstly, it can be shown that $\rho$ converges to $\rho^{\infty}=\max _{[0,1]} \frac{r}{d}$, i.e., to the smallest value $\rho$ such that

$$
r(x)-d(x) \rho \leq 0 \text { on }[0,1]
$$

Secondly, we have concentration in $x$ on a zero-measure set.

Theorem 3.3. (i) $\rho$ converges to $\rho^{\infty}$, the smallest value $\rho$ such that $r(x)-d(x) \rho \leq 0$ on $[0,1]$.

(ii) $n(t, \cdot)$ concentrates on the set $\left\{x \in[0,1], r(x)-d(x) \rho^{\infty}=0\right\}$.

(iii) Furthermore, if this set is reduced to a singleton $x^{\infty}$, then $n(t, \cdot) \rightarrow \rho^{\infty} \delta_{x^{\infty}}$ in $M^{1}(0,1)$, where $M^{1}(0,1)$ is the space of bounded measures on $[0,1]$, dual of the space of continuous functions $C^{0}([0,1], \mathbb{R})$.

Proof of the theorem (1-population case), obtaining simultaneously convergence and concentration by using a Lyapunov function:

Although in the 1D case a direct proof of convergence based on a bounded variation (BV) hypothesis may be obtained, from which concentration easily follows due to the exponential nature of the solutions, it is interesting to note, as this argument can be used in the case of 2 populations, see below Section 3.4, that a global proof based on the design of a Lyapunov function gives at the same time convergence and concentration: choosing 


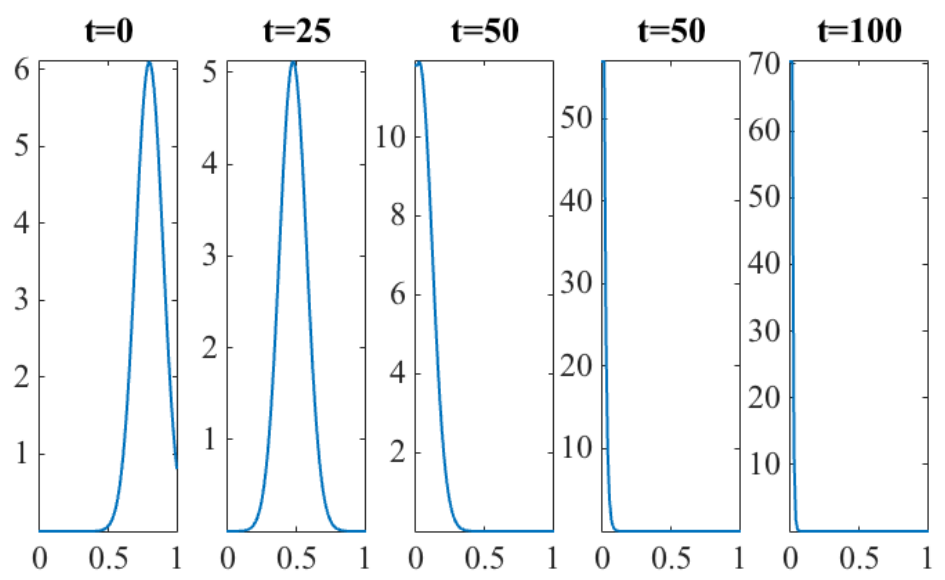

Figure 7: Concentration: plot of the distribution of phenotype $x$ in the population $n(t, x)$ for different times $t$.

any measure $n^{\infty}$ on $[0,1]$ such that

$$
\int_{0}^{1} n^{\infty}(x) d x=\rho^{\infty}=\max _{[0,1]} \frac{r}{d^{\prime}}
$$

and for an appropriate weight $w(x)$ (in fact $1 / d(x))$ setting

$$
V(t)=\int_{0}^{1} w(x)\left\{n(t, x)-n^{\infty}(x)-n^{\infty}(x) \ln n(t, x)\right\} d x,
$$

one can show that

$$
\frac{d V}{d t}=-\left(\rho(t)-\rho^{\infty}\right)^{2}+\int_{0}^{1} w(x)\left\{r(x)-d(x) \rho^{\infty}\right\} n(t, x) d x,
$$

which is always nonpositive, tends to zero for $t \rightarrow \infty$, thus making $V$ a Lyapunov function, and showing at the same time convergence and concentration. Indeed, in this expression, the two terms are nonpositive and their sum tends to zero; the zero limit of the first one accounts for convergence of $\rho(t)$, and the zero limit of the second one accounts for concentration in $x$ (on a zero-measure set) of $\lim _{t \rightarrow+\infty} n(t, x)$.

Note that this kind of nonlocal integrodifferential model can be enriched to become a reaction-diffusion, or reaction-diffusion-advection model, studied numerically, e.g., in $[27,28]$. 


\subsection{Non-local Lotka-Volterra 2D model (2 populations, healthy, $H$, and cancer, C) with 2 different drugs and one resistance phenotype $x$}

A natural extension of the previous nonlocal Lotka-Volterra model to 2 populations, one healthy and one cancerous, leads to the following equations

$$
\begin{aligned}
\frac{\partial}{\partial t} n_{H}(t, x) & =\left[\frac{r_{H}(x)}{1+k_{H} u_{2}(t)}-d_{H}(x) I_{H}(t)-u_{1}(t) \mu_{H}(x)\right] n_{H}(t, x), \\
\frac{\partial}{\partial t} n_{C}(t, x) & =\left[\frac{r_{C}(x)}{1+k_{C} u_{2}(t)}-d_{C}(x) I_{C}(t)-u_{1}(t) \mu_{C}(x)\right] n_{C}(t, x),
\end{aligned}
$$

where we define an 'environment' function standing for the nonlocal logistic term (without added drug): $I_{H}(t)=a_{H H} \cdot \rho_{H}(t)+a_{H C} \cdot \rho_{C}(t), I_{C}(t)=a_{C H} \cdot \rho_{H}(t)+a_{C C} \cdot \rho_{C}(t)$, with $a_{C H}<<$ $a_{C C}$ and $a_{H C}<<a_{H H}$ (intraspecific competition - within the same niche - being always higher than interspecific competition), and $\rho_{H}(t)=\int_{0}^{1} n_{H}(t, x) d x, \rho_{C}(t)=\int_{0}^{1} n_{C}(t, x) d x, u_{1}$ being a cytotoxic drug, $u_{2}$ a cytostatic drug. In this setting, the cytotoxic drug $u_{1}$ acts as an added death term, whereas the cytostatic drug $u_{1}$ only slows down proliferation without killing cells, at least at low doses $\left(u_{1}\right.$ can be thought of as a targeted therapy, e.g., a tyrosine kinase inhibitor).

As a proof of concept, so far without a theorem, an illustration of possible behaviours for the 2 cell populations is shown on Figures 8 and 9. By increasing the drug doses, one can obtain extinction of the cancer cell population while the healthy cell population is preserved.

\subsection{Asymptotic behaviour with constant controls for the nonlocal 2-population case Lotka-Volterra model: a theorem}

Following an argument by P.-E. Jabin \& G. Raoul [24], it can be proved that convergence and concentration occur simultaneously by making use of a Lyapunov functional of the form

$$
\int w(x)\left\{n(t, x)-n^{\infty}(x)-n^{\infty}(x) \ln n(t, x)\right\} d x .
$$

Theorem 3.4. ([39]) (Asymptotic behaviour theorem, generalising to 2 populations the 1D case)

Assume that $u_{1}$ and $u_{2}$ are constant: $u_{1} \equiv \bar{u}_{1}$, and $u_{2} \equiv \bar{u}_{2}$. Then, for any positive initial population of healthy and of tumour cells, $\left(\rho_{H}(t), \rho_{C}(t)\right)$ converges to the equilibrium point $\left(\rho_{H}^{\infty}, \rho_{C}^{\infty}\right)$, which can be exactly computed as follows:

Let $a_{1} \geq 0$ and $a_{2} \geq 0$ be the smallest nonnegative real numbers such that

$$
\frac{r_{H}(x)}{1+\alpha_{H} \bar{u}_{2}}-\bar{u}_{1} \mu_{H}(x) \leq d_{H}(x) a_{1} \quad \text { and } \quad \frac{r_{C}(x)}{1+\alpha_{C} \bar{u}_{2}}-\bar{u}_{1} \mu_{C}(x) \leq d_{C}(x) a_{2} .
$$



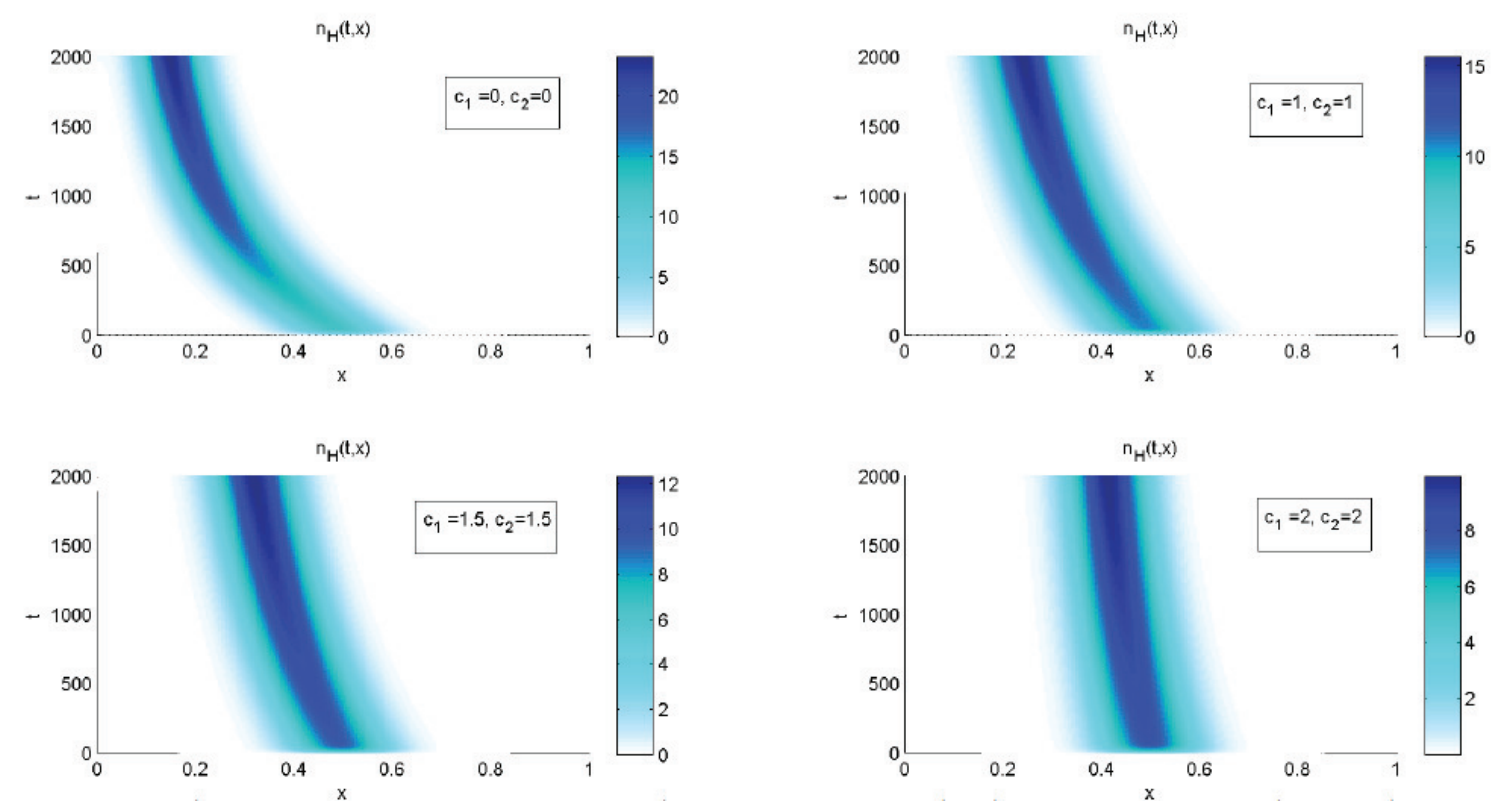

Figure 8: Healthy (or sensitive) cell population case. Simultaneous combinations of 2 drugs, one cytotoxic $\left(u_{1}\right)$ and one cytostatic $\left(u_{2}\right)$, with increasing equal constant doses. Proof of concept, or here "Pedestrian's optimisation", illustrated in the $(x, t)$ plane (resistance phenotype in abscissae and time in ordinates). Increasing constant and equal doses of the 2 drugs, from 0 to $1,1.5$ and 2 (arbitrary units), to be read from left to right, first line of 2 , then second line of 2: healthy cells are preserved. [29].

Then $\left(\rho_{H}^{\infty}, \rho_{C}^{\infty}\right)$ is the unique solution of the invertible $\left(a_{H H} \cdot a_{C C}>>a_{C H} \cdot a_{H C}\right)$ system

$$
I_{H}^{\infty}=a_{H H} \rho_{H}^{\infty}+a_{H C} \rho_{C}^{\infty}=a_{1}, \quad I_{C}^{\infty}=a_{C H} \rho_{H}^{\infty}+a_{C C} \rho_{C}^{\infty}=a_{2} .
$$

Let $A_{H} \subset[0,1]$ (resp., $A_{C} \subset[0,1]$ ) be the set of all points $x \in[0,1]$ such that equality hold in one of the inequalities above. Then the supports of the probability measures

$$
v_{H}(t)=\frac{n_{H}(t, x)}{\rho_{H}(t)} d x \quad \text { and } \quad v_{C}(t)=\frac{n_{C}(t, x)}{\rho_{C}(t)} d x
$$

converge respectively to $A_{H}$ and $A_{C}$ as $t$ tends to $+\infty$.

This theorem, proved by a Lyapunov functional argument (and of which, contrary to the 1D case, we do not know any proof otherwise) can furthermore be generalised to the case of $N>2$ interacting populations [40] (even though those $N$ populations do not represent cancer and healthy cell populations any more), by making use of the same Lyapunov argument.

Note that in the initial framework of 2 populations, healthy and cancer, detailed numerical studies have recently been led in a close, but more general setting by other authors [7]. 

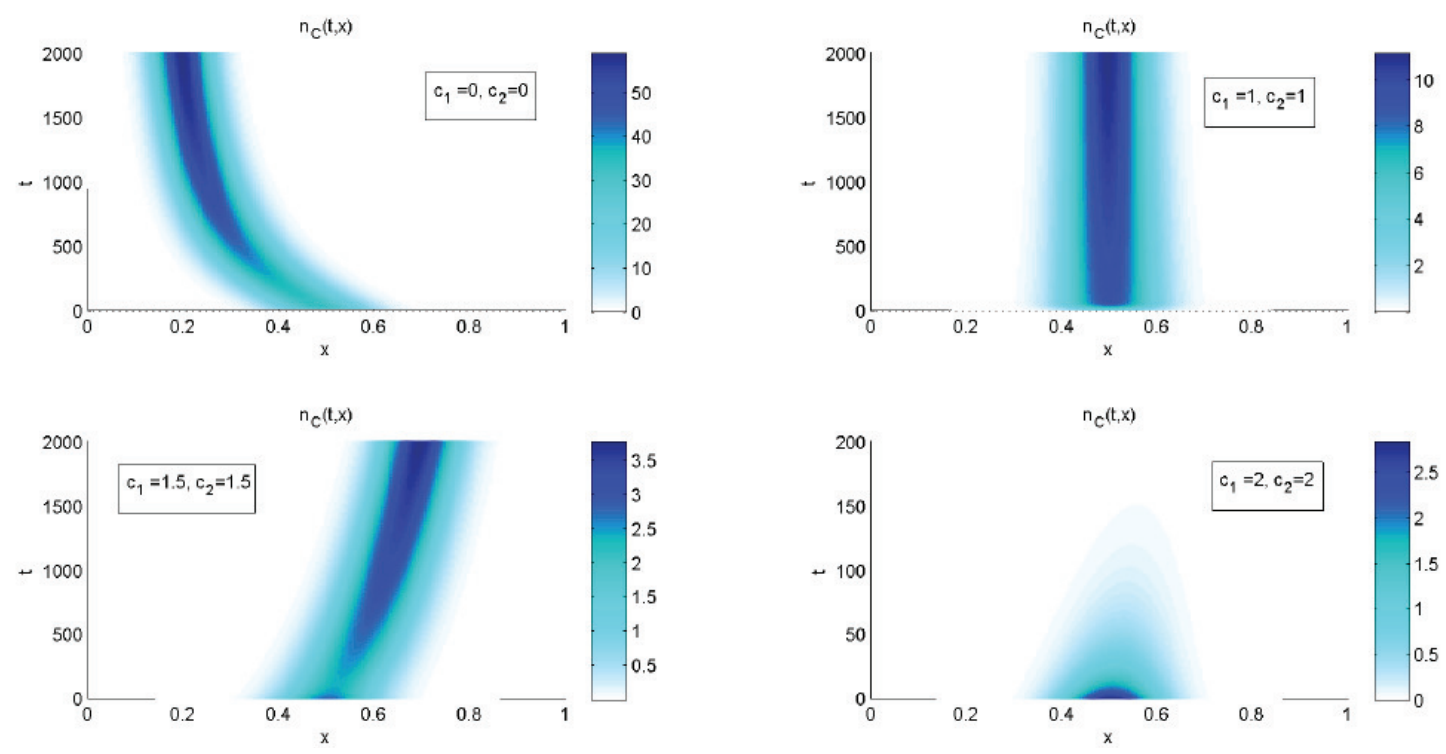

Figure 9: Resistant cancer cell population case. Simultaneous combinations of 2 drugs, one cytotoxic $\left(u_{1}\right)$ and one cytostatic $\left(u_{2}\right)$, with increasing equal constant doses. Proof of concept, or here "Pedestrian's optimisation", illustrated in the $(x, t)$ plane (resistance phenotype in abscissae and time in ordinates). Increasing constant and equal doses of the 2 drugs, from 0 to $1,1.5$ and 2 (arbitrary units), to be read from left to right, first line of 2, then second line of 2: initially as sensitive as healthy cells, cancer cells develop resistance, however to an insufficient level, and eventually go to extinction. A successful example of risky (as shown indeed in Section 4.1) massive drug delivery in a population of cancer cells that are not amenable to develop enough resistance [29].

\section{Therapeutic control}

\subsection{How to be deleterious by using constant and high doses of drugs}

It is a well-known fact in many therapeutic situations, in particular in antibiotherapy and in oncology, that exposing an organism, whose proliferation must be fought, to constant and high doses of drugs is the best way to end up with resistance to the treatment and total therapeutic inefficacy. Figure 10 illustrates on an example a phenomenon indeed common to many therapeutic situations, the fact that the use of massive drug doses in a constant schedule on populations of living organisms with the aim to eradicate them constantly leads to resistance to the drug.

This resistance-inducing killing effect ('What does not kill me strengthens me' [38], paraphrasing Nietzsche) should be avoided as long as possible in therapeutics. In summary (and this will be shown in the next section): limit proliferation but do not try too hard to kill cells, lest the cell population should become resistant, and rather give cytotoxics at high doses (maximum tolerated dose, MTD) during a short interval of time only, thus avoiding to trigger resistance. Note that the cytostatic drug $u_{2}$ only slows down proliferation (softly slowing down velocity in the cell division cycle), but does not arrest it, at least at low doses, whereas the cytotoxic drug $u_{1}$ kills the cells by increasing the death 

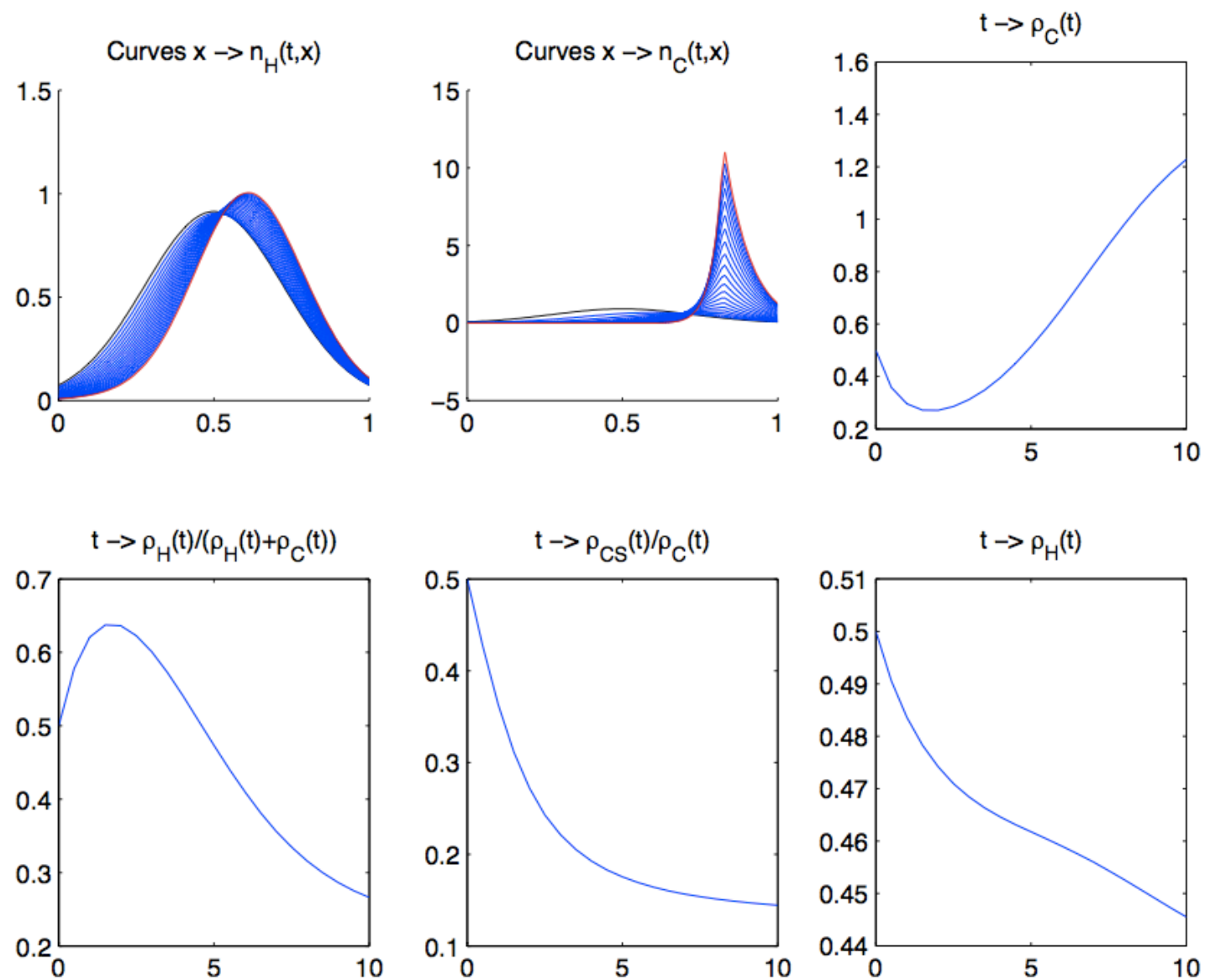

Figure 10: What should never be done (the deleterious therapy): Starting from a Gaussian-like distribution of the resistance phenotype on the whole interval for the two populations, exposed to constant and high dose delivery of the cytotoxic drug, one can observe strikingly different results for its asymptotic distribution: quite small effects of the drug pressure on the phenotype of the healthy (sensitive) cell population $n_{H}$, while the resistant one $n_{C}$ quickly concentrates around a resistant phenotype. First 2 figures, left: distributions of the resistance phenotype $x$ in the 2 populations, healthy (1st) and cancer (2nd). Together with this phenotype concentration effect, one can observe on the other 4 figures the catastrophic effects of this drug delivery strategy on 1) the total cell population of cancer cells $\left.\rho_{C}(t)=\int_{0}^{1} n_{C}(t, x) d x, 2\right)$ the ratio of healthy cells $\rho_{H}$ over the total cell population $\left.\rho_{H}+\rho_{C}, 3\right)$ the population of sensitive cancer cells $\rho_{C S}(t):=\int_{0}^{1}(1-x) n_{C}(t, x) d x$, and 4$)$ the total population of healthy cells $\rho_{H}(t)=\int_{0}^{1} n_{H}(t, x) d x$ [39].

term, hence it is actually a direct life threat to the cell population, that 'defends itself' (by biological mechanisms that are partly known, linked to its plasticity, not represented here).

Also note that an alternative strategy to such use of MTD is metronomic therapy, that seems to prevent the development of resistance by giving low doses of cytotoxics 
over an extended period of time, likely expecting that the population, to some extent thwarted in its proliferation, will be kept in check by the immune system. This has not been represented in our optimal control perspective thus far (however, see [5]).

\subsection{Optimal control problem, 2-population nonlocal Lotka-Volterra model}

The cell environment being defined as in Section 3.3 for each one of the two populations $n_{H}$ and $n_{C}$ as a linear combination of the two total cell populations: $I_{H}(t)=a_{H H} \cdot \rho_{H}(t)+$ $a_{H C} \cdot \rho_{C}(t), I_{C}(t)=a_{C H} \cdot \rho_{H}(t)+a_{C C} \cdot \rho_{C}(t)$, in which coefficients $a$.. represent the intensity of interactions between them (competition being much stronger within a niche than between niches, i.e., $a_{H H}>>a_{H C}$ and $\left.a_{C C}>>a_{C H}\right)$, with again $\rho_{H}(t)=\int_{0}^{1} n_{H}(t, x) d x, \rho_{C}(t)=$ $\int_{0}^{1} n_{C}(t, x) d x$, the optimal control problem for the integro-differential model with evolution in the expression $x$ of a phenotype of resistance to the cytotoxic drug $u_{1}$ reads

$$
\begin{aligned}
\frac{\partial}{\partial t} n_{H}(t, x)= & \left(\frac{r_{H}(x)}{1+\alpha_{H} u_{2}(t)}-d_{H}(x) I_{H}(t)-u_{1}(t) \mu_{H}(x)\right) n_{H}(t, x) \\
\frac{\partial}{\partial t} n_{C}(t, x)= & \left(\frac{r_{C}(x)}{1+\alpha_{C} u_{2}(t)}-d_{C}(x) I_{C}(t)-u_{1}(t) \mu_{C}(x)\right) n_{C}(t, x) \\
& 0 \leq u_{1}(t) \leq u_{1}^{\max }, \quad 0 \leq u_{2}(t) \leq u_{2}^{\max } .
\end{aligned}
$$

Find controls $\left(u_{1}, u_{2}\right)$ minimising

$$
C_{T}\left(u_{1}, u_{2}\right)=\rho_{C}(T)=\int_{0}^{1} n_{C}(T, x) d x,
$$

under the additional constraints

$$
\frac{\rho_{H}(t)}{\rho_{H}(t)+\rho_{C}(t)} \geq \theta_{H C}, \quad \rho_{H}(t) \geq \theta_{H} \cdot \rho_{H}(0),
$$

(the last constraint, with, e.g., $\theta_{H}=0.6$, is here to limit damage to healthy cells).

Theorem 4.1. (Optimal control theorem) ([39]) Under these conditions, the optimal trajectory in large time $T>0$ consists of 2 parts:

- a long-time part, with constant controls on $\left[0, T_{1}\right]$, at the end of which populations have almost concentrated in phenotype (for $T_{1}$ large)

- a short-time part on $\left[T_{1}, T\right]$ consisting of at most three arcs, for $T-T_{1}$ small:

1. a boundary arc, along the constraint $\frac{\rho_{H}(t)}{\rho_{H}(t)+\rho_{C}(t)}=\theta_{H C}$,

2. a free arc (no constraint saturating) with controls $u_{1}=u_{1}^{\max }$ and $u_{2}=u_{2}^{\max }$, 
3. a boundary arc along the constraint $\rho_{H}(t) \geq \theta_{H} \cdot \rho_{H}(0)$ with $u_{2}=u_{2}^{\max }$.

The second short-time phase is all the more efficient as the phenotypes are more concentrated (hence, as the time $T$ is large).

Figure 11 illustrates this theorem by simulations (optimisation using AMPL-IPOPT) for $T=30$ and for $T=60$.

The main idea to interpret this theorem is the following:

1. Let the system naturally evolve to a phenotype concentration (long-time phase).

2. Then, apply the maximal quantity of drugs, during a short-time phase, in order to eradicate as many tumour cells as possible.

Note that this strategy lets the cancer cell population $\rho_{C}$ grow initially to an equilibrium level, while increasing the ratio $\rho_{C S} / \rho_{C}$ of drug-sensitive cancer cells, before delivering $u_{1}=u_{1}^{\max }$; only then is the cytotoxic efficacy maximal.

\subsection{Mixed phenotype-spatial model (simulations without optimal control) [30]}

In this section, we investigate (only by simulations) a model in which, contrary to the previous ones, Cartesian space is represented. Whereas it does not necessarily make sense to set spatial variables to represent a tumour setting where nothing is known of the tumour geometry - and indeed, tumour geometries can be difficult to define, in particular for malignant haemopathies, that are liquid tumours of the bone marrow - spatially structured solid tumours exist, and they frequently adopt at their initial, non vascularised, stage the shape of spheroids. In such tumours, the outer rim is the place where both nutrients (glucose,oxygen) and anticancer drugs are received. Below is proposed such a spatial model, with representation of the effects of different combinations of cytotoxic $\left(u_{1}\right)$ and cytostatic $\left(u_{2}\right)$ drugs, that nevertheless takes into account the issue of drug-induced drug resistance.

Here, both a 1D (radial, for a tumour spheroid) spatial variable $r$ and a resistance phenotype $x$ are considered as structuring a population of cancer cells able to develop resistance. We assume that the evolution of functions $n$ cell population), $s$ (nutrients), $u_{1}$ and $u_{2}$ (drug concentrations) in a $3 \mathrm{D}$ radially symmetric tumour spheroid $(r \in[0,1])$ is ruled by the following set of equations:

$$
\begin{array}{r}
\partial_{t} n(t, r, x)=\left[\frac{p(x)}{1+\mu_{2} u_{2}(t, r)} s(t, r)-d \varrho(t, r)-\mu_{1}(x) u_{1}(t, r)\right] n(t, r, x), \\
-\sigma_{s} \Delta s(t, r)+\left[\gamma_{s}+\int_{0}^{1} p(x) n(t, r, x) d x\right] s(t, r)=0,
\end{array}
$$



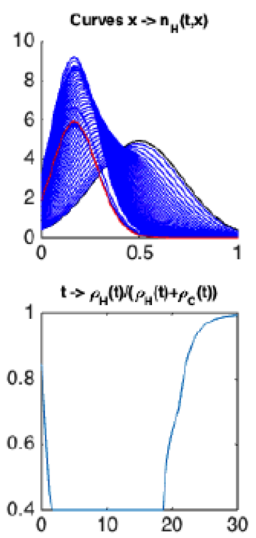

$t \rightarrow u_{1}(t)$
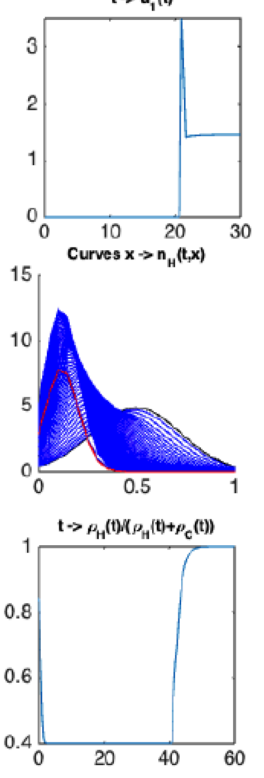

$t \rightarrow u_{1}(t)$

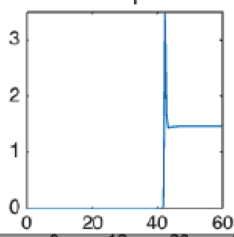

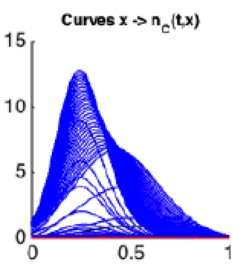

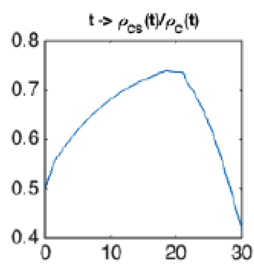

$t \rightarrow u_{2}(t)$
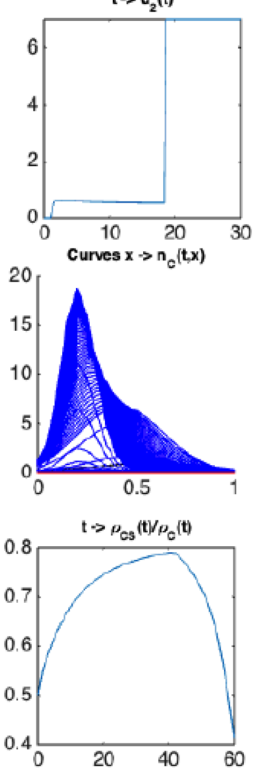

$t \rightarrow \mathrm{U}_{2}(\mathrm{t}$

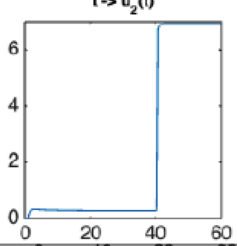

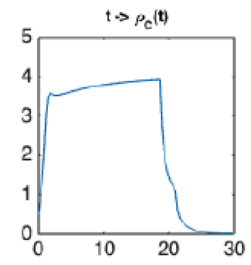

$t \rightarrow \rho_{H}(t)$
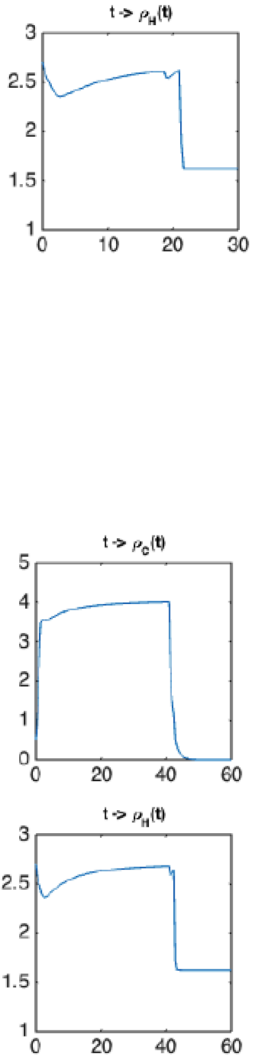

Figure 11: Solution to the optimal control problem for $T=30$ (upper eight panels) and for $T=60$ (lower eight panels). Same sequence of 6 first figures as in Figure 10, plus optimal control solutions for cytotoxic $u_{1}$ and cytostatic $u_{2}$ (last 2 figures). Note that the optimal control strategy lets the cancer cell population $\rho_{C}$ grow initially to an equilibrium level, while increasing the ratio $\frac{\rho_{C S}}{\rho_{C}}$ of drug-sensitive cancer cells, before delivering $u_{1}=u_{1}^{\max }$; only then is the cytotoxic treatment efficacy maximal. The optimal control trajectory for the cytotoxic drug is made of 3 parts, the first one with $u_{1}=0$, the 2 nd one with $u_{1}=u_{1}^{\max }$, the 3 rd one with $u_{1}$ slightly lower than $u_{1}^{\max }$ [39]. The horizon $T$ is fixed, and the switching time $T_{1}$ changes with $T$, but the behaviour of the solutions is the same, whatever the value of $T$. 


$$
\begin{aligned}
& -\sigma_{u} \Delta u_{1}(t, r)+\left[\gamma_{u}+\int_{0}^{1} \mu_{1}(x) n(t, r, x) d x\right] u_{1}(t, r)=0, \\
& -\sigma_{u} \Delta u_{2}(t, r)+\left[\gamma_{u}+\mu_{2} \int_{0}^{1} n(t, r, x) d x\right] u_{1}(t, r)=0
\end{aligned}
$$

with zero Neumann conditions at $r=0$ coming from radial symmetry and Dirichlet boundary conditions at $r=1$

$$
s(t, r=1)=s_{1}, \quad \partial_{r} s(t, r=0)=0, \quad u_{1,2}(t, r=1)=u_{1,2}(t), \quad \partial_{r} u_{1,2}(t, r=0)=0 .
$$

In these equations, the first one describing proliferation of the cancer cell population, the other three representing diffusion in space, from the rim $r=1$ toward the center $r=0$ of the spheroid, of a nutrient $s$ (standing for oxygen, glucose, fatty acids, etc., addressing proliferation) and of the two drugs $u_{1}$ and $u_{2}$ (addressing the added death rate due to the drugs), the proliferation function $p$ and the drug sensitivity functions $\mu_{1}$ have been chosen to be simple decreasing affine functions of the resistance phenotype $x$ : $p(x):=a_{1}+a_{2}(1-x)$ and $\mu_{1}(x)=b_{1}+b_{2}(1-x)\left(\mu_{2}\right.$ being a sensitivity parameter to cytostatic infusion is taken constant, not inducing resistance, contrary to the cytotoxic sensitivity $\mu_{1}(x)$, whereby resistance occurs).

For each $t$, we also define

$$
\left.\rho(t, r)=\int_{0}^{1} n(t, r, x) d x \text { (local density at radius } r\right)
$$

and

$$
\rho_{T}(t)=\int_{0}^{1} \rho(t, r) r^{2} d r \text { (global density) }
$$

The initial distribution of the cell population is illustrated on Figure 12. Its evolution under constant doses of cytostatic or cytotoxic drugs is illustrated on Figure 13, and under combined doses of bang-bang and constant controls on Figure 14.

The simulation illustrated by Figure 14 shows on an example (as a proof of concept) that in periodic drug delivery strategies, one should never give cytotoxics on a constant scheme, but that a bang-bang delivery of cytotoxics may be successful. This is in accordance with the theoretical results of the previous Sections 4.1 and 4.2.

\section{Conclusion: Why is evolution important in cancer?}

In conclusion, we provide a list of challenging, though basic, questions on multicellularity and cancer:

- (Admitted) cancer is a disease of multicellular organisms, that has been evidenced, including in fossils, in the whole animal kingdom. 

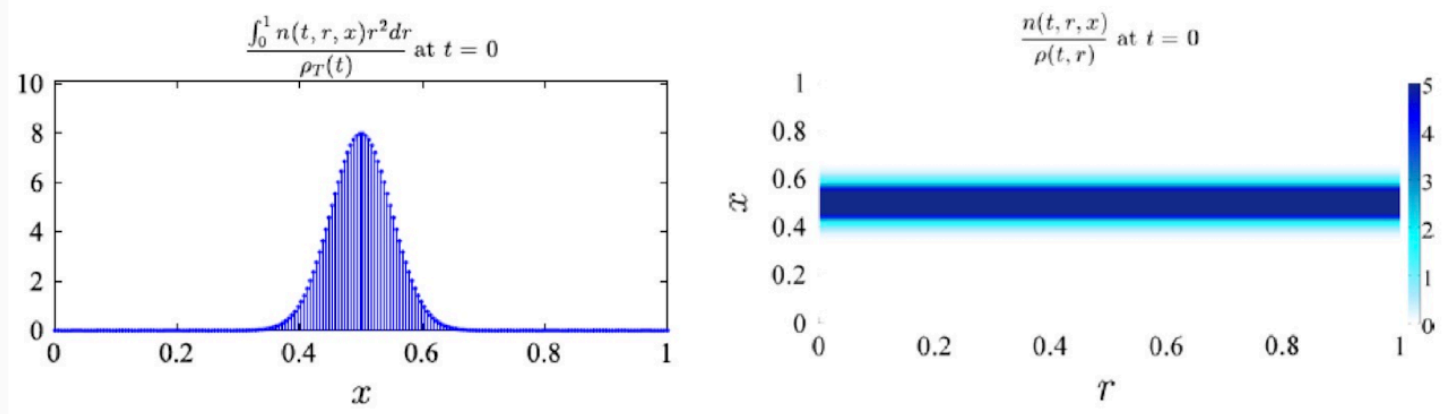

Figure 12: Initial distribution of the cell population and its representation in the $(r, x)$ plane (phenotype $x$ arbitrarily set to 0.5 to follow its trend under different conditions, see Figure 13).

- (Admitted) cancer is the failure of maintenance of a coherent (=founded on stable cellular differentiations) multicellularity, or else:

- (Admitted) cancer may be defined as a loss of cohesion of tissues and organs of a same organism following failures in differentiation.

- Does there exist in the construction of multicellularity a qualitative succession (major transitions) of emergences of families of genes responsible for 1. proliferation and apoptosis 2. differentiation (transcription factors?); 3. epigenetic control of differentiations? In favour on this scenario, phylogenetic scenarios of evolution of mutations in acute myeloid leukaemia (AML) go in the opposite direction with increasing malignancy [19].

- Some gene mutations predispose subjects to well-identified organ cancers: do these genes play a role in the anatomic constitution of multicellularity?

- Evolution proceeds by tinkering [25], using every possible avaible material: what in such a succession of tinkerings makes an organism viable but fragile?

- The genes that are altered in cancers are the same that serve multicellularity design $[10,12,13,52]$ : can we methodically collect these genes and their emergence, so as to better understand the development of cancers?

- What defines a same organism ? A 'self' that would be conserved during the sequences of differentiations that in Man lead from the first embryonic cell to the '200 terminally differentiated cell types'?

- What holds together, normally without conflict, the cell types (the interferon pathways??), and what does the immune system recognise as non-self (foe rather than friend) in a cancer cell? 
(a)

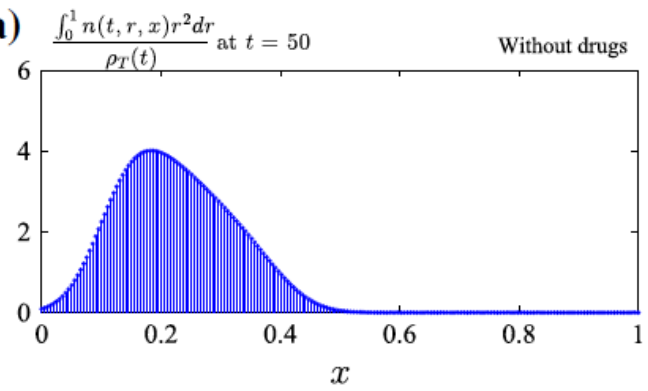

(b) $\underline{\int_{0}^{1} n(t, r, x) r^{2} d r}$ at $t=200$

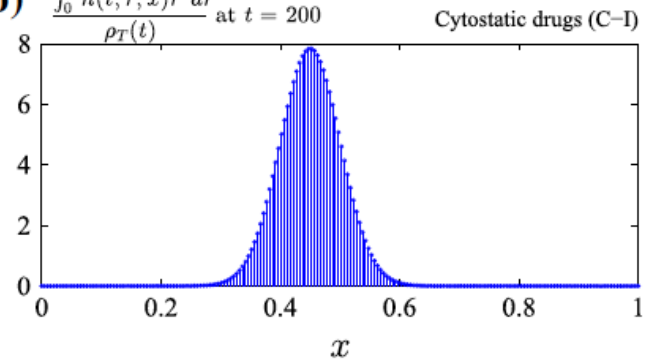

(c) $\underline{\int_{0}^{1} n(t, r, x) r^{2} d r}$ at $t=120$

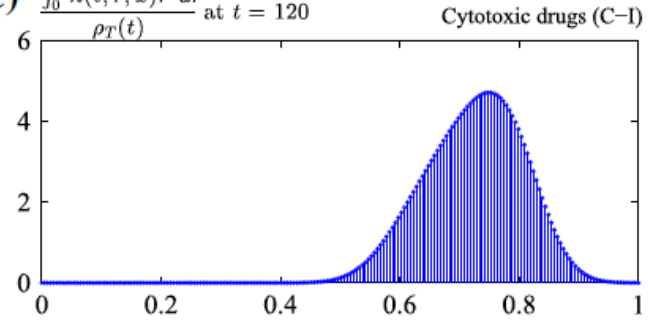

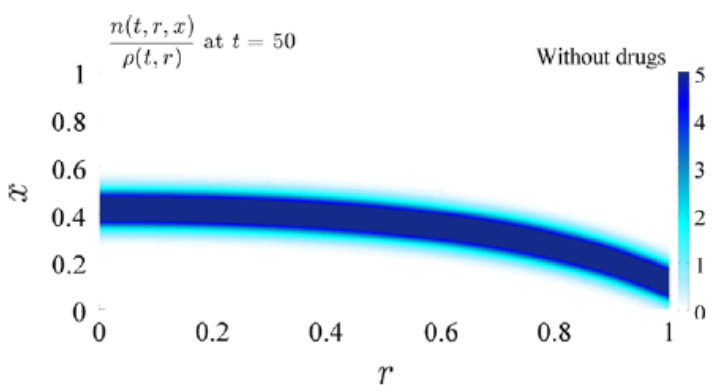
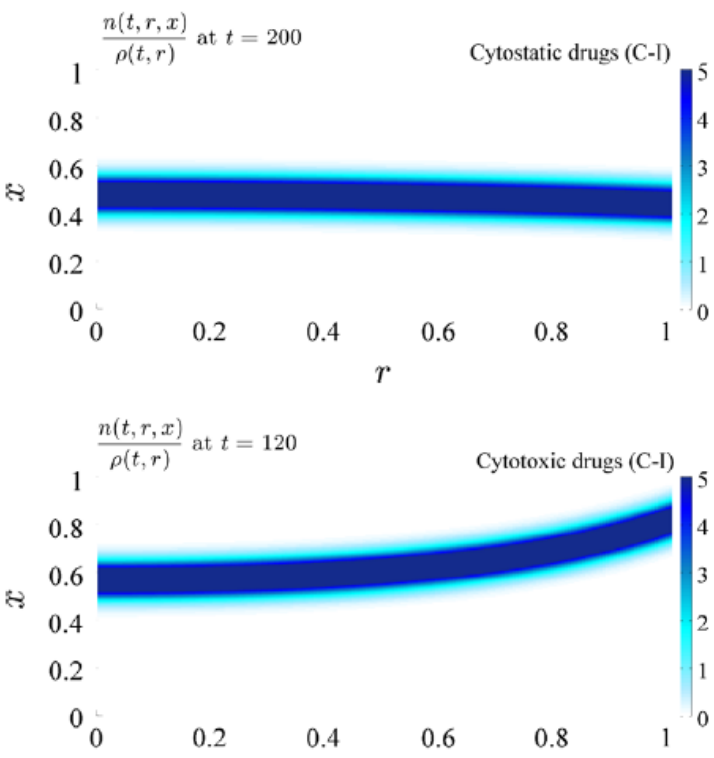

Figure 13: Long-time evolution of phenotype $x$ without drugs (a), under exposure to cytostatics (b) and to cytotoxics (c) constant infusion (C-I). On the right panels, $x$ is in ordinates and $r$, from 0 (centre of the spheroid) to 1 (outer rim of the spheroid), in abscissae. One can see that without drugs, the phenotypes slowly reverts towards sensitivity, that the cytostatic drug $u_{2}$ has only small effects, whereas the cytotoxic drug $u_{1}$ induces resistance [30].

- Is there a relationship of such coherence with the major histocompatibility complex (MHC)? What is its primary function, if not to ensure organism cohesion (of tissues), and how does such coherence (of signals) operate?

- Can we parallel evolution of species and evolution of their immune system? Some enlightenment to collect genes active at multicellularity constitution?

- Admitting that loss of control of cell differentiations is the main cause of cancer, can we speculate that all cancers have in their evolution an epigenetic origin or an epigenetic mandatory step?

- Some is known of mutations in genes that control epigenetics (e.g., DNMT3A, 

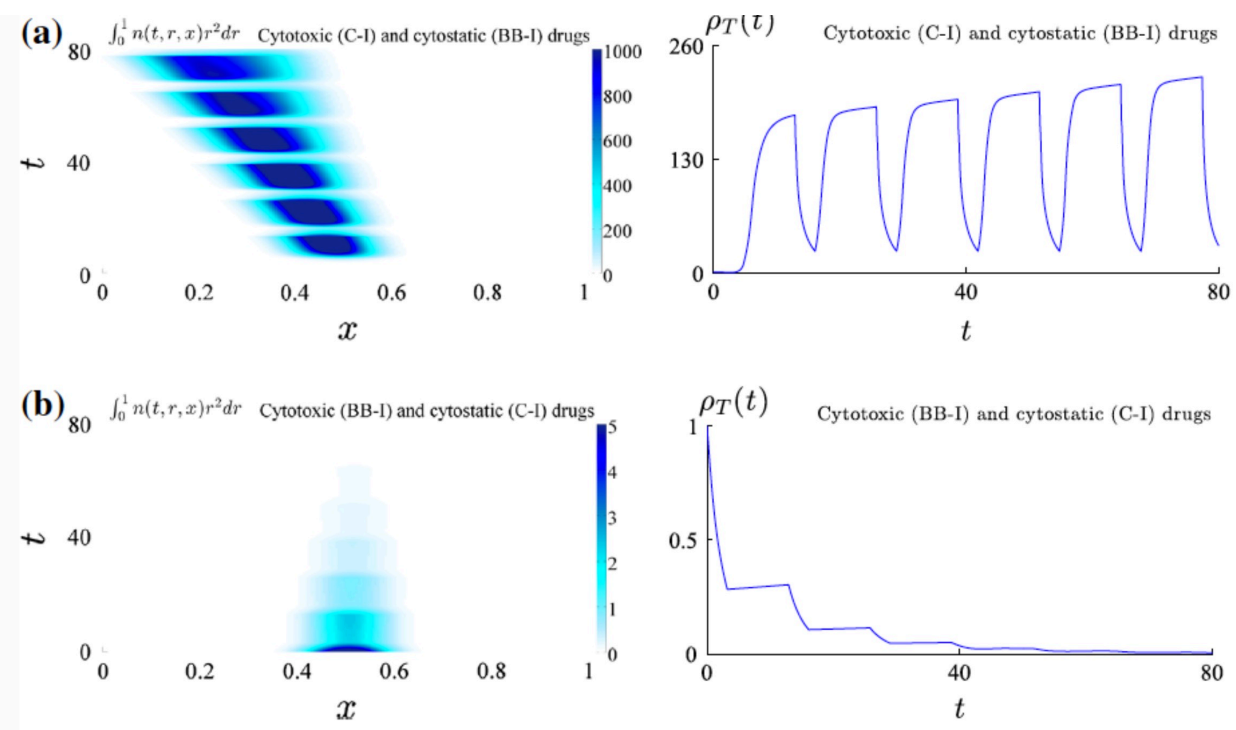

Figure 14: (a) Cytotoxic (constant infusion, C-I) with cytostatic (bang-bang infusion, BB-I) drug infusions. Plots of $\int_{0}^{1} n(t, r, x) r^{2} d r$ in the $(x, t)$ plane (left panel) and $\rho_{T}(t)$ with time $t$ (right panel). Bang-bang infusion of cytostatic drugs together with constant infusion of cytotoxic drugs weakly affects the dynamics of cancer cells by comparison with the case without therapies, apart from temporary reductions of the global population density. (b) Cytotoxic (BB-I) with cytostatic (C-I) drug infusions. Plots of $\int_{0}^{1} n(t, r, x) r^{2} d r$ (left panel) and $\rho_{T}(t)$ (right panel). Bang-bang infusion of cytotoxic drugs together with constant delivery of cytostatic drugs can push the cancer cell population toward extinction. The time unit is days. All values are normalised with respect to the initial global population density [30]. This example shows that in a periodic drug delivery framework, strategy (b) should clearly be better than strategy (a).

TET2) in early leukaemogenesis, and of genes of cell metabolism (IDH1, IDH2) in cancers (AML, glioblastoma): can we propose and exemplify a standard scenario linking perturbations of metabolism / perturbations of epigenetic control of differentiations / cancers?

- The energetic metabolism of the cell involves intercellular communications, that have been reported to be disrupted in cancer: is the emergence of gap junctions essential in the development of multicellularity and are perturbations of physiological gap junctions a cause or a consequence of cancer in solid tumours? [45-47].

- Cancer cell populations seem to be able to switch between glycolytic and mitochondrial respiratory phenotypes: do cancer cells shift easily from one to the other? In other words, does a tumour practice a form of metabolic bet hedging?) [20].

- What are the advantages and drawbacks of these 2 phenotypes, glycolytic and respiratory? Efficiency of the tricarboxylic acid [TCA, aka Krebs] cycle vs. rapidity of anaerobic glycolysis? When did the mitochondrial respiratory chain appear 
in evolution as a necessary condition for the establishment of reliable intercellular communications?

- What is more relevant for stress response of a cell population (adaptable, as in the case of a tumour): maintain a subpopulation of all-stress resistant cells, or maintain a subpopulation of cells expressing 'cold genes' and able to launch different resistance mechanisms in different cells? (... stochastically chosen?)

- Can bet hedging be seen as a 'tumour strategy' to diversify the responses of a plastic cell population to deadly stress (as high doses of cytotoxic drugs) by launching different stress response mechanisms in different cells? (ABC transporters, detoxication enzymes, blocking influx, DNA repair, and others) [3].

- Does stress response induce derepression of cold genes? Presented in [50], this hypothesis proposes that the existence of very ancient genes, constituted in a remote past of our planet, makes plastic cell populations able to put at work different survival programs in a state of emergency, with bet hedging, i.e., (stochastic or not) distribution of survival strategies, in a cancer cell population.

- Does bet hedging shuffle phenotypes, setting favorable bases for the emergence of specialisation [1,32-35] and cooperativity in tumours [31,36, 42], making them viable?

\section{Acknowledgments}

The author would like to thank Tommaso Lorenzi, Alexander Lorz, Benoît Perthame, Camille Pouchol and Emmanuel Trélat, co-authors of the mathematical papers whose main results are presented in this study.

\section{References}

[1] C.A. Aktipis, A.M. Boddy, R.A. Gatenby and et al. Life history trade-offs in cancer evolution. Nature Rev. Cancer, 13:883-892, 2013.

[2] T. Boveri. The origins of malignant tumors. Williams Wilkins, Baltimore, 1929.

[3] B. Brutovsky and D. Horvath. Structure of intratumor heterogeneity: is cancer hedging its bets? arXiv (2013),1307.0607.

[4] K.J. Bussey, L.H. Cisneros, C.H. Lineweaver and et al. Ancestral gene regulatory networks drive cancer. PNAS, 114(24):6160-6162, 2017.

[5] C. Carrère. Optimization of an in vitro chemotherapy to avoid resistant tumours. J. Theor. Biol., 413:24-33, 2017.

[6] R.H. Chisholm, T. Lorenzi and J. Clairambault. Cell population heterogeneity and evolution towards drug resistance in cancer: biological and mathematical assessment, theoretical treatment optimisation. Biochem. Biophys. Acta, 1860:2627-2645, 2016.

[7] H. Cho and D. Levy. The impact of competition between cancer cells and healthy cells on optimal drug delivery. Math. Mod. Nat. Phenom., 2019, to appear. 
[8] L.H. Cisneros, K.J. Bussey, A.H. Orr and et al. Ancient genes establish stress-induced mutation as a hallmark of cancer. PLoS One, 12(4):e0176258, 2017.

[9] A.S. Cleary, T.L. Leonard, S.A. Gestl and et al. Tumour cell heterogeneitymaintained by cooperating subclones in Wnt-driven mammary cancers. Nature Lett., 508:113-117, 2014.

[10] P.C.W. Davies and C.H. Lineweaver. Cancer tumors as metazoa 1.0: tapping genes of ancient ancestors. Phys. Biol., 8(1):015001, 2011.

[11] T. Dobzhansky. Nothing in biology makes sense except in the light of evolution. American Biology Teacher, 35(3):125-129, 1973.

[12] T. Domazet-Lošo and D. Tautz. An ancient evolutionary origin of genes associated with human genetic diseases. Mol. Biol. Evol., 25(12):2699-2707, 2008.

[13] T. Domazet-Lošo and D. Tautz. Phylostratigraphic tracking of cancer genes suggests a link to the emergence of multicellularity in metazoa. BMC Biol., 8(1):66, 2010.

[14] R.J. Gillies, D. Verduzco and R.A. Gatenby. Evolutionary dynamics of carcinogenesis and why targeted therapy does not work. Nat. Rev. Cancer, 12(7): 487-493, 2012.

[15] A. Goldman, M Kohandel and J. Clairambault. Integrating biological and mathematical models to explain and overcome drug resistance in cancer, Part 1: biological facts and studies in drug resistance. Current Stem Cell Reports, 3:253-259, 2017.

[16] A. Goldman, M. Kohandel and J. Clairambault. Integrating biological and mathematical models to explain and overcome drug resistance in cancer, Part 2: from theoretical biology to mathematical models. Current Stem Cell Reports, 3:260-268, 2017.

[17] M.M. Gottesman. Mechanisms of cancer drug resistance. Annu. Rev. Med., 53: 615-627, 2002.

[18] M.M. Gottesman, T. Fojo and S.E. Bates. Multidrug resistance in cancer: role of ATPdependent transporters. Nat. Rev. Cancer, 2(1): 48-58, 2002.

[19] C.A. Gravenmier, M. Siddique and R.A. Gatenby. Adaptation to stochastic temporal variations in intratumoral blood flow: the warburg effect as a bet hedging strategy. Bull Math Biol, 80(5):954-970, 2017.

[20] P. Hirsch, Y. Zhang, R. Tang and et al. Genetic hierarchy and temporal variegation in the clonal history of acute myeloid leukaemia. Nat. Commun, 7:12475, 2016.

[21] S. Huang. On the intrinsic inevitability of cancer: From foetal to fatal attraction. Sem. Canc. Biol., 21:183-199, 2011.

[22] S. Huang. Genetic and non-genetic instability in tumor progression: link between the fitness landscape and the epigenetic landscape of cancer cells. Canc. Metastasis Rev., 32:423-448, 2013.

[23] L. Israel. Tumour progression: random mutations or an integrated survival response to cellular stress conserved from unicellular organisms? J. Theor. Biol., 178(4):375-380, 1996.

[24] P.E. Jabin and G. Raoul. On selection dynamics for competitive interactions. J. Math. Biol., 63:493-551, 2011.

[25] F. Jacob. Evolution and tinkering. Science , 196 (4295):1161-1166, 1977.

[26] C.H. Lineweaver, P.C.W. Davies, M.D. Vincent and et al. Targeting cancers̈ weaknesses (not its strengths): therapeutic strategies suggested by the atavistic model. Bioessays, 36(9):827835, 2014.

[27] T. Lorenzi, R.H. Chisholm, L. Desvillettes and et al. Dissecting thedynamics of epigenetic changes in phenotype-structured populations exposed to fluctuating environments. J. Theor. Biol., 386:166-176, 2015.

[28] T. Lorenzi, R.H. Chisholm, J. Clairambault and et al. Tracking the evolution of cancer cell populations through the mathematical lens of phenotype-structured equations. Biology Direct, 11:43, 2016. 
[29] A. Lorz, T. Lorenzi, M.E. Hochberg and et al. Populational adaptive evolution, chemotherapeutic resistance and multiple anti-cancer therapies. ESAIM Math. Model. Numer. Anal., 47(2):377-399, 2013.

[30] A. Lorz, T. Lorenzi, J. Clairambault and et al. Modeling the effects of space structure and combination therapies on phenotypic heterogeneity and drug resistance in solid tumors. Bull. Math. Biol., 77(1):1-22, 2014.

[31] A. Marusyk, V. Almendro and K. Polyak. Intra-tumour heterogeneity: a looking glass for cancer? Nature Rev. Cancer 12: 323-334, 2012.

[32] J. Maynard Smith and E. Szathmáry. The major transitions in evolution. W.H. Freeman, Publisher, Oxford, 1995.

[33] R.E. Michod and D. Roze. Cooperation and conflict in the evolution of multicellularity. Heredity, 36:1-7, 2001.

[34] R.E. Michod and D. Roze. Cooperation and conflict during evolutionary transitions in individuality. J. Evol. Biol., 19:1406-1409, 2006.

[35] R.E. Michod, Y. Viossat, C.A. Solari and et al. Life-history evolution and the origin of multicellularity. J. Theor. Biol., 239:257-272, 2006.

[36] K. Polyak and A. Marusyk. Clonal cooperation. Nature, 508:52-53, 2014.

[37] A.O. Pisco, A. Brock, J. Zhou and et al. Non-Darwinian dynamics in therapy-induced cancer drug resistance. Nat. Commun., 4:2467, 2013.

[38] A.O. Pisco and S. Huang. Non-genetic cancer cell plasticity and therapy-induced stemness in tumour relapse: 'What does not kill me strengthens me'. Br. J. Cancer, 112(11):1725-32, 2015.

[39] C. Pouchol, J. Clairmbault, A. Lorz and et al. Asymptotic analysis and optimal control of an integro-differential system modelling healthy and cancer cells exposed to chemotherapy. J. Math. Pures Appl., 116:268-308, 2018.

[40] C. Pouchol and E. Trélat. Global stability with selection in integrodifferential Lotka-Volterra systems modelling trait-structured populations. J. Biol. Dynamics, 12(1):872-893, 2018.

[41] S.V. Sharma, D.Y. Lee, B. Li and et al. A chromatin-mediated reversible drug-tolerant state in cancer cell subpopulations. Cell, 141:69-80, 2010.

[42] D.P. Tabassum and K. Polyak. Tumorigenesis: it takes a village. Nature Rev. Cancer , 15:473483, 2015.

[43] A.S. Trigos, R.B. Pearson, A.T. Papenfuss and et al. Altered interactions between unicellular and multicellular genes drive hallmarks of transformation in a diverse range of solid tumors. PNAS, 114 (24):6406-6411, 2017.

[44] A.S. Trigos, R.B. Pearson, A.T. Papenfuss and et al. How the evolution of multicellularity set the stage for cancer. Br. J. Cancer, 118:145-152, 2018.

[45] J.E. Trosko. Mechanisms of tumor promotion: possible role of inhibited intercellular communication. Eur. J. Cancer Clin. Oncol., 23(6):599-601, 1987.

[46] J.E. Trosko. Gap junctional intercellular communication as a biological "Rosetta stone" in understanding, in a systems biological manner, stem cell behavior, mechanisms of Epigenetic toxicology, chemoprevention and chemotherapy. J. Membrane Biol., 218:93-100, 2007.

[47] J.E. Trosko. A conceptual integration of extra-, intra- and gap junctional-intercellular communication in the evolution of multi-cellularity and stem cells: how disrupted cell-cell communication during development can affect diseases later in life. Int. J. Stem Cell Res. Ther., 3(1):021, 2016.

[48] M.D. Vincent. Cancer: a de-repression of a default survival program common to all cells?: a life-history perspective on the nature of cancer. Bioessays, 34(1):72-82, 2011. 
[49] C.H. Waddington. The strategies of the genes. George Allen E Unwin, London, 1957.

[50] A. Wu, Q. Zhang, G. Lambert and et al. Ancient hot and cold genes and chemotherapy resistance emergence. Proc. Nat. Acad. Sci., USA, 112:10467-10472, 2015.

[51] J.S. You and P.A. Jones. Cancer genetics and epigenetics: two sides of the same coin? Cancer Cell, 22(1):9-20, 2012.

[52] J.XW. Zhou, L. Cisneros, T. Knijnenburg and et al. Phylostratigraphic analysis of tumor and developmental transcriptomes reveals relationship between oncogenesis, phylogenesis and ontogenesis. Converg. Sci. Phys. Oncol., 4:025002, 2018. 